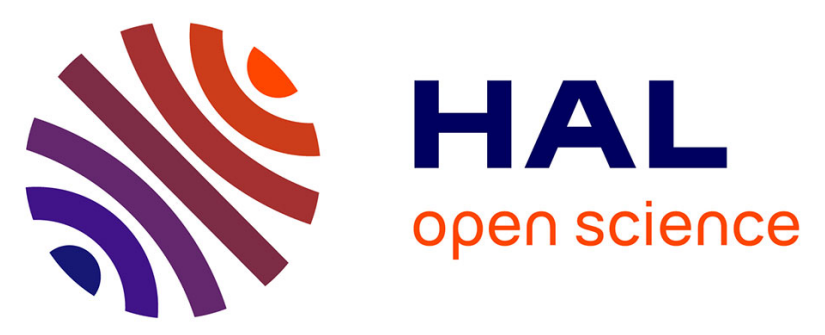

\title{
Cambodge. Compte rendu de la réunion scientifique internationale de Siem Reap. Journées de travail sur Angkor (21-23 mars 1994)
}

Christine Hawixbrock

\section{- To cite this version:}

Christine Hawixbrock. Cambodge. Compte rendu de la réunion scientifique internationale de Siem Reap. Journées de travail sur Angkor (21-23 mars 1994). Bulletin de l'Ecole française d'ExtrêmeOrient, 1994, 81 (1), pp.360 - 363. 10.3406/befeo.1994.2266 . halshs-02549205

\section{HAL Id: halshs-02549205 \\ https://shs.hal.science/halshs-02549205}

Submitted on 21 Apr 2020

HAL is a multi-disciplinary open access archive for the deposit and dissemination of scientific research documents, whether they are published or not. The documents may come from teaching and research institutions in France or abroad, or from public or private research centers.
L'archive ouverte pluridisciplinaire HAL, est destinée au dépôt et à la diffusion de documents scientifiques de niveau recherche, publiés ou non, émanant des établissements d'enseignement et de recherche français ou étrangers, des laboratoires publics ou privés. 


\section{Cambodge. Compte rendu de la réunion scientifique internationale} de Siem Reap. Journées de travail sur Angkor (21-23 mars 1994)

Christine Hawixbrock

\section{Citer ce document / Cite this document :}

Hawixbrock Christine. Cambodge. Compte rendu de la réunion scientifique internationale de Siem Reap. Journées de travail sur Angkor (21-23 mars 1994). In: Bulletin de l'Ecole française d'Extrême-Orient. Tome 81, 1994. pp. 360-363;

doi : https://doi.org/10.3406/befeo.1994.2266

https://www.persee.fr/doc/befeo_0336-1519_1994_num_81_1_2266

Fichier pdf généré le 08/02/2019 


\title{
CAMBODGE
}

\author{
Compte rendu de la réunion scientifique internationale de Siem Reap \\ Journées de travail sur Angkor (21-23 mars 1994)
}

Dans le cadre d'une ouverture pluridisciplinaire concernant les recherches sur l'ancien pays khmer, trois journées de travail sur Angkor se sont tenues à Siem Reap, du 21 au 23 mars 1994, grâce à l'initiative conjointe de l'École française d'Extrême-Orient et de l'Université Sophia de Tokyo, avec le soutien du ministère cambodgien de la Culture.

Une trentaine de chercheurs scientifiques spécialisés en archéologie, épigraphie, géologie, hydrographie, restauration architecturale, sciences de l'environnement et en botanique - cambodgiens, français, japonais et indonésiens ${ }^{9}$ - se sont réunis sur le site d'Angkor pour confronter leurs projets de recherches et les résultats des travaux en cours, pour permettre une meilleure circulation des informations scientifiques et définir des objectifs communs dans l'élaboration de projets futurs. Pour la première fois peut-être dans l'histoire de la recherche au Cambodge, le dialogue s'est enfin ouvert entre les diverses institutions et les nombreuses sciences intéressées à améliorer les connaissances encore bien imprécises dans nombre de domaines, sur les époques préangkoriennes et angkoriennes et sur les liens historiques qu'elles ont entretenu avec le monde indianisé du Sud-Est asiatique et des pays d'Extrême-Orient.

\section{Programme d'activité de ces journées}

Une première prise de contact entre les différents participants a eu lieu l'après-midi du 21 dans les locaux du Grand Hôtel de Siem Reap, au cours de laquelle chacun a pu exposer dans sa langue les points principaux de ses recherches en cours et de ses projets, grâce aux efforts combinés des coordinateurs de cette réunion, MM. P. Souyri (directeur des études de l'EFEO) et Y. Tsuboi (professeur à l'Université de Hokkaido), qui ont assumé les traductions en français et en japonais. M. Ang Choulean assurait la traduction français-khmer, et M. Lao Kim Leang assurait la traduction japonais-khmer.

De manière à conserver un lien étroit avec le terrain de l'étude, matériau primordial pour chacun des chercheurs présents et à favoriser les échanges « sur le vif», les deux journées suivantes se sont déroulées sur les sites archéologiques. Les participants se sont partagés entre quatre groupes interactifs mettant chacun en valeur un aspect particulier de la recherche, ainsi définis : Groupe I : Architecture et restauration ; Groupe II : Archéologie et histoire ; Groupe III : Histoire, beaux-arts et patrimoine ; Groupe IV : Espace et environnement (habitat, forêt et hydrologie). Ces quatre groupes ont été organisés de manière que chaque participant soit libre d'évoluer à l'intérieur de chacun d'entre eux, et je me dois de dire ici qu'il fut parfois bien difficile à beaucoup d'entre nous de choisir à quel groupe se rattacher, tant les thèmes abordés présentaient des aspects qui pouvaient intéresser de près ou de loin nos disciplines personnelles.

\section{Aperçu des sites et des thèmes abordés}

De ce fait, nous nous sommes tous rejoints le matin du 22 mars autour du Pr Nakao Yoshiharu sur le chantier archéologique de Banteay Kdei, implanté à l'intérieur de la troisième enceinte du temple, sur le front nord de la «salle aux Danseuses » et de l'édifice à piliers. M. Nakao a pu ainsi nous montrer les vestiges du soubassement en latérite d'un édifice rectangulaire antérieur au temple de Banteay Kdei, dégagé par son équipe, et nous exposer les pre-

9. Voir la liste des participants en annexe 1. 
mières conclusions de ses fouilles : le bâtiment mis au jour semble nettement antérieur à Banteay Kdei (édifié sur ordre du roi Jayavarman VII à la fin du XII ${ }^{e}$ siècle), il porte en bordure du soubassement les marques de trous de poteaux, qui pourraient justifier une élévation en bois mais, son dégagement n'étant pas terminé, les résultats des campagnes de fouilles suivantes permettront probablement de déterminer la destination de cet édifice et sa date exacte. MM. B. Dagens et J. Dumarçay ont émis l'hypothèse qu'il pourrait s'agir de vestiges appartenant au site de Kuti, fondé par Jayavarman II au IX ${ }^{\mathrm{e}}$ siècle et mentionné dans les stèles de Bat Chum et de Tep Pranam, site que George Cœdès proposait de situer sur l'emplacement même de Banteay Kdei où des piédroits inscrits en réemploi mentionnent l'érection de divinités à Kutisvara.

Les problèmes de conservation des sites ont ensuite été abordés, toujours à Banteay Kdei, autour de Yokoyama Jun, botaniste chargé de déterminé quels arbres sont susceptibles de devenir un danger pour les temples par l'ampleur de leur taille et de leurs racines. Les conclusions de ses recherches l'ont amené à proposer la mise en place d'un périmètre de sécurité défriché autour des édifices et enceintes des monuments, de manière à protéger ces derniers, tout en gardant par ailleurs les grands arbres dans tous les autres endroits hors risque, de façon à conserver ombrage et caractère à tous ces sites, implantés dans l'actuel parc forestier d'Angkor.

Puis, ce fut Jacques Dumarçay, responsable de la restauration des monuments d'Angkor pour l'EFEO, qui nous guida sur le chantier de restauration de la Terrasse du Roi lépreux (fin XII ${ }^{\mathrm{e}}$ siècle), chantier commencé à la fin des années 60 par le conservateur d'Angkor, Bernard Philippe Groslier et qui avait dû s'interrompre au début des années 70 . Rouvert en 1993, ce chantier qui a pour but de remettre en place les bas-reliefs du parement extérieur de la terrasse a été confié à deux jeunes architectes, Christophe Pottier et Pascal Royère. Nous avons pu alors observer les techniques utilisées pour replacer les blocs couverts de reliefs avant de nous déplacer sur le site tout proche du temple-montagne du Baphuon (milieu du $\mathrm{XI}^{\mathrm{e}}$ siècle), prochain site à restaurer prévu par J. Dumarçay, également abandonné dans les années 70 . Nous avons pu avec lui, tout en visitant le temple, réfléchir sur la complexité de la restauration architecturale appliquée à des temples angkoriens ayant subi des modifications au cours de leur histoire et sur les techniques a mettre en œuvre à ce propos. C'est le cas notamment de ce monument (le Baphuon), sur lequel un Buddha couché monumental avait été édifié sur la face ouest de la pyramide à une époque relativement tardive, et pour la construction duquel la galerie du premier étage avait presque totalement été démantelée, servant ainsi de réserve de blocs de construction. Implanté au niveau du second étage du Baphuon, ce Buddha avait englobé dans sa structure la galerie du second étage de la pyramide, couverte de bas-reliefs. La restauration de ce monument est par ailleurs particulièrement importante à trois titres, d'abord pour des raisons historiques, puisqu'il constitue le centre de la seconde Angkor et le temple d'État du roi Udayadityavarman II, ensuite sur le plan de l'histoire de l'art khmer, puisqu'il est le seul temple à Angkor possédant sur plusieurs de ses étages et enceintes des bas-reliefs à scènes religieuses et astrologiques traitées en panneaux au niveau des baies et des fenêtres, enfin au niveau technique, puisque ce chantier devrait permettre d'utiliser des méthodes jamais tentées en Asie. La préparation du travail se fera par infographie, la construction d'une voûte en béton armé placée sous le Buddha couché, permettra la circulation à l'intérieur de la seconde galerie masquée (reconstruite par récupération des blocs utilisés pour le Buddha couché) avant la réédification dudit Buddha, pour lequel de nouveaux blocs de grès seront taillés, et qu'il serait ainsi possible de conserver comme témoin de la longue vie religieuse de ce temple.

L'après-midi du 22 mars, deux groupes se sont constitués, le groupe " Architecture et Restauration » se rendant à Angkor Vat pour discuter des meilleures possibilités d'intervention pour la restauration de la chaussée d'accès d'Angkor Vat qui enjambe les douves du temple, tandis que le groupe «Espace et Environnement» se rendait d'abord sur les abords de la rivière de Siem Reap pour étudier les systèmes d'irrigation des rizières et des vergers par l'utilisation de norias en bambou, survivance peut-être des moyens d'irrigation utilisés aux époques anciennes, puis sur le Tonlé Sap, avec la visite du village lacustre des pêcheurs, excellent exemple ethno-archéologique d'un village se déplaçant de toutes pièces plusieurs fois 
dans l'année suivant les saisons, en fonction de la hausse ou de la baisse des eaux du Grand Lac. Cette visite permit également de réfléchir ensemble sur l'existence possible d'un port à l'époque d'Angkor à un emplacement similaire - hypothèse émise par Christophe Pottier - et sur les éventuelles traces archéologiques qu'il aurait laissées. Nous supposons personnellement que la réalité actuelle du village migrant pourrait fort bien recouvrir une réalité ancienne qui laisse peu de chances (étant donné également le haut taux d'envasement du lac) de découvrir les vestiges archéologiques d'un port périodiquement démonté pour toujours rester situé dans un niveau d'eau suffisant, nécessaire à l'ancrage des bateaux.

La première partie de la matinée du 23 mars s'est déroulée dans les locaux de l'EFEO de Siem Reap, et a consisté pour chacun des groupes à mettre en commun réflexions et informations diverses de façon à envisager une action scientifique commune à Angkor sur tous les thèmes abordés. Puis les participants se divisèrent pour étudier, d'une part, les problématiques de fouilles envisagées par l'EFEO en 1995 au Palais royal d'Angkor Thom, d'autre part, de nouvelles problématiques de restauration architecturale autour des temples de Thommanon, Chau Say Thevoda et Ta Kev, et enfin pour étudier l'hydraulique, l'environnement des baray ainsi que les réseaux hydrauliques qui en résultent autour du baray occidental, immense réservoir d'eau artificiel construit probablement au début du $\mathrm{XI}^{\mathrm{e}}$ siècle. Nous avons pu à cette occasion visiter le temple d'Ak Yum, monument préangkorien situé à l'intérieur d'une ville partiellement disparue sous le baray occidental, entièrement dégagé nouvellement, et ce pour la première fois depuis le début des travaux effectués à Angkor à l'aube du $\mathrm{XX}^{\mathrm{e}}$ siècle. Il nous fut ainsi possible de l'étudier en détail, montrant ainsi, s'il en était besoin, que le travail scientifique et les recherches historiques à Angkor ont une longue histoire devant eux et nécessiteront le concours des nombreux spécialistes internationaux intéressés à l'étude de ce pays, au patrimoine culturel exceptionnel.

L'après-midi du 23 mars nous permit, grâce à l'obligeance de $M$. le ministre Vann Molyvann et du conservateur, de découvrir les dépôts archéologiques de la Conservation d'Angkor, à l'intérieur desquels quelque 5000 à 6000 pièces (dont essentiellement des statues), sont conservées. Ce dépôt eut à subir, il y a peu de temps, des vols que l'on ne peut que déplorer. L'inventaire exhaustif de l'ensemble des pièces débutera en juin 1994, assorti d'une publication prévue pour $1997^{10}$.

Ces trois journées de travail se sont conclues dans les locaux du Grand Hôtel de Siem Reap, lors d'une séance plénière au cours de laquelle chacun des délégués a pu proposer des projets dans l'optiques de travaux communs. Les voix furent unanimes pour apprécier l'intérêt de cette réunion et plébisciter de nouvelles rencontres de ce type, dont la seconde est prévue pour l'année 1995. Les interventions de clôture ont été prononcées par MM. Denys Lombard et Ishizawa Yoshiaki.

\section{Annexe $1:$ Liste des participants par ordre alphabétique (nationalités confondues)}

Hasan Ambary, directeur du service archéologique indonésien ; Ros Borath, architecte, conseiller du ministre de la Culture du gouvernement royal du Cambodge ; Patrick Cernesson, compagnon tailleur de pierre, Monuments historiques ; Ang Choulean, ethnologue, chercheur à l'EFEO, Université des beaux arts de Phnom Penh ; Bruno Dagens, archéologue, ancien membre de l'EFEO, Université de Paris III, Sorbonne nouvelle ; Jacques Dumarçay, architecte, ancien membre de l'EFEO ; Christian Dupavillon, architecte, chargé de mission interministériel pour la coordination au Cambodge ; Endo Nobuo, ingénieur des sites, Institut des cultures asiatiques, Université de Sophia ; Jacques Gaucher, architecte, membre de l'EFEO, Université d'architecture de Nantes ; Christine Hawixbrock, archéologue attachée à l'EFEO ; Ishizawa Yoshiaki, Institut des cultures asiatiques, Université de Sophia; Ito Nobuo, architecte, ancien directeur de Tokyo International ; Katagiri Masao, architecte, Université de Tezukayama-Gakuin ; Lao Kim Leang, spécialiste sur l'environnement, Institut de recherches

10. Mission dirigée par Bruno Dagens, à Paris, et par Christine Hawixbrock, à Siem Reap. 
sur l'environnement ; Denys Lombard, directeur de l'EFEO ; Miyakawa Asaichi, hydrologue, chargé de recherche auprès du ministère de la Construction ; Moriai Tomio, géologue, Institut Tohoku de technologie ; Nakao Tomio, archéologue, Université de Tezukayama-Gakuin ; Nishimura Yukio, architecte, faculté de technologie, Université de Tokyo ; Christophe Pottier, architecte, responsable de l'EFEO à Siem Reap ; Pascal Royère, architecte, attaché à l'EFEO ; Long Seam, Institut orientaliste de Moscou, Université de Phnom Penh ; Shigeeda Yutaka, architecte, Collège des sciences et de technologie, Université Nichidai ; Pierre Souyri, directeur des études à l'EFEO ; Tsuboi Yoshiharu, Université de Hokkaido ; Vann Molyvann, architecte, ministre de la Culture du gouvernement royal du Cambodge ; Yokohama Jun, botaniste, Université de Tokyo ; Oung Von, directeur de la Conservation d'Angkor.

\section{Annexe 2 : Liste des rapports d'activité et projets écrits}

Rapports d'activité et projets de l'équipe japonaise (par ordre alphabétique)

Endo Nobuo: Activités communes et activités individuelles dans la coopération culturelle internationale.

Ito Nobuo : Related to the Conservation of Angkor Monuments.

Katagiri Masao : Fundamental policy for the restoration of the Angkor Monuments.

Moriai Tomio : Geological research on the monuments and ground of Angkor, study of latérite.

Nakao Yoshiharu : First excavations of Angkor Monuments.

Nishimura Yukio : Sukhothai historical park development project and its impact on local communities.

Shigeeda Yutaka : Architectural surveys conducted at the Banteay Kdei monument.

Tsukawaki Shinji : A geological approach to interpreting the Khmer civilisation.

Ueno Kunikazu : Wat Phu in Laos and Khmer civilisation.

Yokohama Jun : Vegetation of the Angkor region and the feasibility of preserving it.

Rapports d'activité et projets de l'EFEO (par ordre alphabétique)

Jacques Dumarçay : Reprise des travaux sur le chantier du Baphuon.

Jacques Gaucher et Christine Hawixbrock: Histoire et archéologie du monde khmer, architectures, villes, territoires et sociétés.

Christophe Pottier et Pascal Royère : Rapport des activités de restauration et Rapport d'activité relatif aux travaux de maintenance à Angkor.

Christophe Pottier : Étude du Prasat Bat Chum.

Pascal Royère : Étude du Khléang Nord. 